\title{
How to trade electricity flexibility using artificial intelligence - An integrated algorithmic framework
}

\author{
Lisa Hanny \\ FIM Research Center, University of Augsburg \\ Project Group Business \& Information \\ Systems Engineering of the Fraunhofer FIT \\ lisa.hanny@fim-rc.de \\ Christina Leinauer \\ FIM Research Center, University of Bayreuth \\ Project Group Business \& Information \\ Systems Engineering of the Fraunhofer FIT \\ Bavarian Center for Battery Technology (BayBatt) \\ christina.leinauer@fit.fraunhofer.de \\ Jens Strueker \\ FIM Research Center, University of Bayreuth \\ Project Group Business \& Information \\ Systems Engineering of the Fraunhofer FIT \\ jens.strueker@fim-rc.de \\ Jan Weissflog \\ FIM Research Center, University of Augsburg \\ Project Group Business \& Information \\ Systems Engineering of the Fraunhofer FIT \\ jan.weissflog@fim-rc.de
}

\begin{abstract}
In course of the energy transition, the growing share of Renewable Energy Sources (RES) makes electricity generation more decentralized and intermittent. This increases the relevance of exploiting flexibility potentials that help balancing intermittent RES supply and demand and, thus, contribute to overall system resilience. Digital technologies, in the form of automated trading algorithms, may considerably contribute to flexibility exploitation, as they enable faster and more accurate market interactions. In this paper, we develop an integrated algorithmic framework that finds an optimal trading strategy for flexibility on multiple markets. Hence, our work supports the trading of flexibility in a multi-market environment that results in enhanced market integration and harmonization of economically traded and physically delivered electricity, which finally promotes resilience in highly complex electricity systems.
\end{abstract}

\author{
Marc-Fabian Körner \\ FIM Research Center, University of Bayreuth \\ Project Group Business \& Information \\ Systems Engineering of the Fraunhofer FIT \\ marc-fabian.k.koerner@uni-bayreuth.de \\ Anne Michaelis \\ FIM Research Center, University of Bayreuth \\ Project Group Business \& Information \\ Systems Engineering of the Fraunhofer FIT \\ anne.michaelis@fim-rc.de \\ Martin Weibelzahl \\ FIM Research Center, University of Bayreuth \\ Project Group Business \& Information \\ Systems Engineering of the Fraunhofer FIT \\ martin.weibelzahl@fim-rc.de
}

\section{Introduction}

In course of the energy transition, the growing share of Renewable Energy Sources (RES) makes electricity generation more decentralized and highly intermittent, resulting in various challenges for system resilience [1]. This requires different flexibility options that make electricity systems future-proof and resilient given an increasingly intermittent RES feed-in. Literature describes the need for an increase in flexibility as the "flexibility gap" [2]. In leveraging the necessary flexibility potentials and closing this gap, digital technologies play a crucial role, as they facilitate information exchange among electricity market participants and make actual flexibility needs more transparent [3, 4]. Moreover, digital technologies enable faster (market) interactions and automated trading close to real-time [3]. As a result, digital technologies and corresponding trading algorithms may exploit flexibility potentials with higher speed and better 
temporal and spatial accuracy than human traders and, thereby, contribute to overall system resilience.

Currently, however, the potential of digital technologies in electricity trading and the corresponding exploitation of flexibility potentials is not fully realized. Even though, algorithmic trading based on Artificial Intelligence (AI) can considerably raise market efficiency, e.g., by increasing market transparency, only about half of the trades on European spot markets are carried out automatically via algorithms [5]. Against this background, algorithmic trading on electricity markets clearly lags behind financial markets [6]. Moreover, only few electricity trading algorithms actively take into account flexibility exploitation, leading to (partially) decoupled electricity and flexibility trading and, ultimately, a mismatch of traded electricity and physical system needs. Even though, some first algorithmic solutions exist for flexibility exploitation, they are mostly restricted to (passive) decision-support and do not actively participate in electricity markets like, e.g., in [7, 8, 9]. Autonomously trading flexibility on wholesale electricity markets as well as flexibility markets would allow algorithms to learn trading strategies that simultaneously maximize profit on multiple markets (for electricity and flexibility) and, thus, foster enhanced market integration and overall system efficiency. Moreover, current approaches to flexibility trading are mainly based on single-market optimizations, for which it becomes increasingly difficult to cope with the complexity of close to real-time, multi-market settings. Thus, standard optimization models may lead to inferior flexibility marketing solutions as compared to AI algorithms that actively learn flexibility marketing strategies by directly interacting and experimenting with the respective market environment.

In this paper, we contribute to existing research by developing an integrated Machine Learning (ML) framework that incorporates flexibility trading on wholesale electricity markets as well as flexibility markets and copes with the challenges of an RES-based electricity system. Against this background, we address the following research questions: What is an applicable categorization scheme for existing electricity and flexibility trading algorithms? What is a suitable design for a ML framework that simultaneously trades different flexibility options across multiple markets? Augmenting existing research on ML-based electricity transactions, our approach allows us, first, to identify current concepts for decentralized flexibility trading from the perspective of individual market participants (instead of electricity system operators). Second, this allows us to derive an appropriate ML framework that is able to handle different, partially unknown, market environments and the time dependency of providing flexibility. Building upon prior work in the combined fields of ML, electricity trading, and flexibility (e.g., [10, $11,12])$, we augment existing research by introducing a multi-market environment with partially unknown market characteristics and different flexibility products in terms of, e.g., time discretization, bid size, speed of availability, etc. To the best of our knowledge, we are the first to propose a holistic algorithmic framework that handles the highly complex and large-scale problem of flexibility trading.

We proceed by providing important background information on electricity and flexibility trading as well as resilience of electricity systems in Section 2. We make use of a systematic literature review to identify and cluster existing algorithmic frameworks for electricity and flexibility trading (Section 3 \& Section 4). Subsequently, we present an integrated ML framework for multi-market flexibility trading (Section 5 ) and illustrate directions for future research (Section 6).

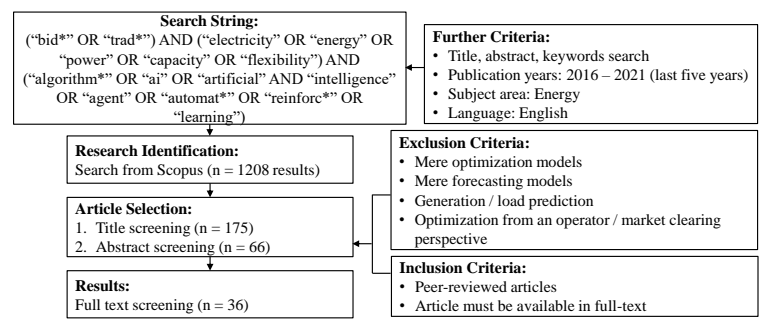

Figure 1: Overview of Literature Review

\section{Background}

As we aim to provide a ML framework for multi-market flexibility trading, this section gives a brief overview about the process of trading on electricity markets as well as a definition of flexibility and flexibility markets.

Trading on wholesale electricity markets generally follows three stages. First, in the bidding stage, Generation companies (GENCOs), electricity suppliers, and electricity consumers place their bids according to their marginal costs, respective, willingness to pay. Second, in the market clearing stage, the market clearing price, given total electricity demand and supply, is set. Third, the last stage is the physical delivery of electricity by the GENCOs [13]. However, these steps may differ and may be adapted when using, e.g., different clearing mechanisms. As our proposed ML framework targets flexibility trading, we focus on the bidding stage of 
electricity trading.

Further, we establish a definition of flexibility for the remainder of this paper. From the flexibility supply perspective, flexibility is defined as a deviation from an ex-ante planned baseline electricity generation or load profile $[14,15]$. From an electricity system perspective, flexibility is needed to balance the demand and supply of electricity at every point in time [16]. Literature knows the following five flexibility options: (1) supply-side flexibility, (2) storage flexibility, (3) transmission flexibility, (4) demand-side flexibility, and (5) inter-sectoral flexibility [17]. As flexibility can be used for different services, from congestion management to frequency control, a number of different flexibility markets exists. Flexibility can be traded at an (inter-)national level to provide system and grid stability, among others. Moreover, flexibility can also be used on a local level to counteract regional imbalances [18]. To realize market-side benefits, flexibility can also be traded on wholesale electricity markets. These different markets all have their own entry barriers and product characteristics such as pre-qualification requirements or minimum bidding volumes. This results in a complex environment where a flexibility provider has to consider numerous combinations of flexibility products on multiple markets.

To handle these complexities and to provide faster - close to real-time - flexibility trading, Information System (IS) tools within the AI field can be used. These tools can help a flexibility provider, but also the electricity system, to ensure a (market-based) optimal use of flexibility, which in turn can improve electricity system resilience.

\section{Methodology}

We conduct a systematic literature review following the guidelines of [19] and [20] to identify the body of knowledge regarding existing algorithmic frameworks for trading on electricity and flexibility markets. In Figure 1 we depict the entire process of our systematic literature review. Regarding the database for our search, we choose Scopus. We derive a search string and complement the relevant topics related to our research question with synonyms. A connection of the topics is established by boolean operators. This results in the following search string: ("bid*" OR "trad*") AND ("electricity" OR "energy" OR "power" OR "capacity" OR "flexibility") AND ("algorithm*" OR "ai" OR "artificial” AND "intelligence" OR "agent" OR "automat*" OR "reinforc*" OR "learning"). For our systematic literature review, we consider the last five years, covering the period from 2016 to 2021, to account for the fast-moving nature of this emerging field of research. For the year 2021, we include papers that are available on Scopus until May, 2021. Besides, we involve only articles written in English language. Regarding the subject area, we specify via a filter option on Scopus to solely consider articles with a focus on energy, since our goal of the systematic literature review is to identify algorithms for electricity trading.

We apply the search string to the title, abstract, and keywords, obtaining 1.208 articles as a result of our initial search. To further narrow down the number of eligible articles, we proceed with the article selection process, which consists of three steps: title screening, abstract screening, and full text screening. In each of these steps we refer to defined exclusion and inclusion criteria: First, we exclude mere optimization models and mere prediction models. Next, we eliminate algorithms that optimize from the point of view of an operator/market clearing authority rather than from the point of view of a market participant. As inclusion criteria, we define that exclusively papers for which a full text is available are taken into account. A further inclusion criterion ensures that only peer-reviewed papers are considered in our review.

As a first step of the selection process, we screen the titles and reduce the number of papers to 175 , for which we additionally screen the abstract. After abstract screening, 66 papers remain for full text screening. As a result of the full text screening, we obtain 36 articles that are relevant to our first research question. To structure the individual algorithmic approaches identified for trading on electricity and flexibility markets, we follow the concept-centric organization of results as proposed by [19]. Therefore, we develop a concept matrix listing the identified articles from the literature review and the identified content assorted to concepts and corresponding units of analysis. Hence, for each article, we analyze which groups of algorithms and approaches for trading electricity and flexibility were identified in the respective article. We use the following concepts to classify the results from the literature review: Type of algorithm, considered electricity market, involved players/participants, algorithm objective, and data input. The classification was carried out by a joint discussion of the authors. With regard to the type of algorithm, we follow the classification of [21] for AI approaches for energy demand response.

\section{Overview of algorithmic approaches for electricity and flexibility trading}

In the following, we will present the results of our literature review of algorithmic approaches 
for electricity and flexibility trading. Although there already exist manifold optimization models for trading, respectively bidding, optimization models have difficulties coping with the complexity of interactive trading in close to real-time markets like, e.g., in $[22,23,24]$. Further, AI-based approaches allow for learning viable solutions without having to derive abstractions for the representation of the real-world [25]. Given this focus on algorithmic approaches for electricity and flexibility trading, we present the identified publications that propose trading algorithms for electricity or flexibility grouped by the used algorithmic approaches. For each category, we describe the identified players, respectively market participants, as well as the considered electricity market(s). Figure 2 shows our proposed classification of all identified algorithmic approaches for electricity and flexibility trading. The individual classification of a publication indicates the applied type of algorithm. In case of an overlap, the respective authors combined several algorithm types.

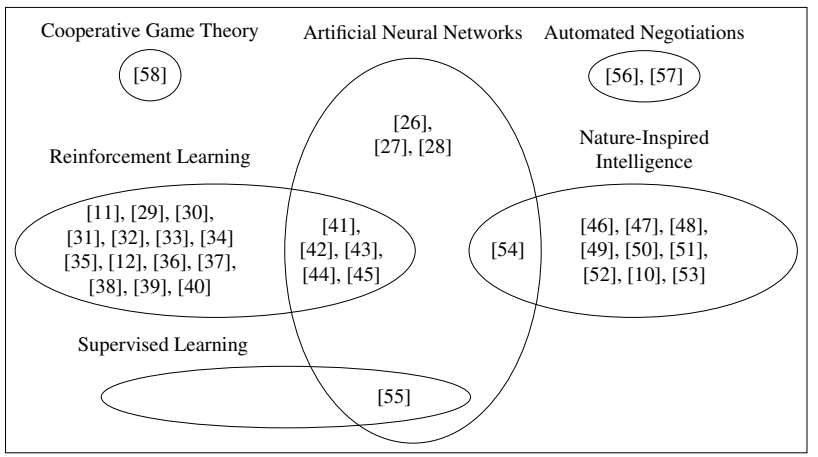

Figure 2: Overview of algorithmic approaches for electricity and flexibility trading

The largest part of existing research on electricity trading or flexibility trading relates to Reinforcement Learning $(R L)$, where $\mathrm{RL}$ is a $\mathrm{ML}$ approach that focuses on learning from the interaction of a specific agent with its environment. The agent learns to improve its actions with respect to a given goal [59]. [11] employs several Q-learning agents, i.e., electricity consumers that contribute to an overall learning strategy, maximizing the consumers' profits. In doing so, the authors include a retail, a wholesale, and a balancing market. [29] also develops a machine learning algorithm for bidding on multiple markets, i.e., the day-ahead and the real-time electricity market. In contrast to previous publications, their approach bases on dynamic programming and the authors compute their results from the perspective of virtual financial entities that do not physically trade their electricity. Similarly, [34] propose a polynomial-time online learning algorithm for virtual trading on the day-ahead and the real-time electricity market. [30] propose a Q-learning approach for a double auction mechanism for trading between several micro-grid operators. Hence, the focus of this approach is more on bilateral trading rather than the application of trading strategies on a specific electricity market. [32] also propose a Q-learning approach. The agents within the Q-learning algorithm of the authors are not micro-grid operators, but micro-grid investors that want to maximize the long-term profit of their respective micro-grids. [33] present an algorithmic approach for trading between micro-grids. In the environment of a Peer-to-Peer (P2P) market, the micro-grids' objective is to learn a strategy that maximizes the financial reward for the prosumers of the respective micro-grids. In contrast to [30], [33] propose a dynamic programming algorithm. [44] and [45] combine RL approaches with Artificial Neural Networks (ANNs). Their deep RL algorithms likewise consider a P2P market environment where the operators of micro-grids trade electricity with each other. Several algorithmic approaches focus on automating the processes that implement bidding strategies on electricity markets from the perspective of electricity suppliers, i.e., GENCOs. [31] propose a Q-learning approach to maximize the profit from selling electricity on the day-ahead market and on a futures market. Similarly, [36] present a RL approach for wind electricity generators on a day-ahead and a subsequent balancing market. [40] also consider bidding strategies on multiple markets, in their case, a day-ahead and a subsequent reserve market. The authors use an eligibility traces algorithm, similar to Q-learning. [35] explore a RL approach with a hybrid stochastic model coupled with a Stackelberg game for bidding on a day-ahead market. [39] employ a RL approach with a Roth-Erev learning strategy to improve the bidding of GENCOs on a wholesale electricity market. Further, [41] and [43] apply a deep deterministic policy gradient algorithm, i.e., an actor-critic approach, to combine the functionalities of RL and ANN, maximizing profits of electricity suppliers by selling their generated electricity. [12] encompass both sides of a double-sided auction, i.e., the selling as well as the buying agents. In order to model the trade between electricity sellers and buyers, the authors apply a Q-learning algorithm. [38] and [42] focus on trading strategies for prosumers, e.g., electricity storage facilities. In contrast, [38] apply RL with a policy function approximation to an intraday-market. [42] apply deep Q-learning in a P2P environment. [37] implement a RL algorithm with multiple agents, representing multiple market participant roles, i.e., prosumers and consumers, on a 
local electricity market.

Besides RL, supervised, and unsupervised learning represent other groups of ML algorithms that detect patterns from data inputs either given a certain output (supervised) or not (unsupervised) [21]. Within our literature review, we have identified one publication that combines supervised learning, more specifically Bayesian networks, with ANNs: [55] analyze the behavior of electricity suppliers within a day-ahead and an intraday market.

By means of our literature review, we identify various approaches relying on Nature-Inspired Intelligence (NII). These approaches take natural processes, e.g., the evolution of individuals within a population or biological swarms, and develop a search algorithm to iteratively find the optimal or near-optimal solution [60]. The identified publications for electricity trading illustrate the range of existing nature-inspired algorithms. [46], [52], [10], [51], and [49] provide a NII approach to derive the best bidding strategy as an electricity supplier on a day-ahead market. [52] and [10] provide a Genetic Algorithm (GA). [49] combines a GA with a particle swarm algorithm. [46] further applies a GA based on a hybrid particle swarm optimization and an improved firefly algorithm. [51] evaluates various NII where a differential evolution and vortex search algorithm perform best. [48] also searches for an optimal bidding strategy from the perspective of electricity suppliers. However, the authors further include a reserve market besides a electricity spot market like a day-ahead market, where they propose an extended gravitational search algorithm. [50] and [47] evaluate NII within a local electricity market environment where $\mathrm{P} 2 \mathrm{P}$ trading is conducted between the market participants. While [50] applies an ant colony optimization, [47] analyze different solution approaches to a bi-level problem, including, amongst others, an ant colony optimization, a hybrid-adaptive differential evolution, and an algorithm vortex search. [54] combine NII with ANNs to derive a bidding strategy for electricity suppliers as well as large consumers. With this approach, the authors deploy an artificial bee colony algorithm and use an ANN to optimize the parameters of the algorithm. [53] combine particle swarm and GAs in order to establish optimal smart contracts for demand response on a blockchain-based platform for electric vehicles.

Even though, some of the publications presented have combined algorithmic approaches like RL or NII with Artificial Neural Networks (ANNs) to improve the outcome of individual approaches, several publications base solely on ANNs. ANNs are a specific form of algorithms inspired by the human nervous system. Since the basic structure and framework of ANNs differ from classic NII like evolutionary algorithms, we present these publications in a different category. [26] use an ANN to maximize the profit of participants of a day-ahead market. [28] apply a feed-forward ANN for the participants of the Iberian Electricity Market. [27] propose a dynamic ANN to support electricity market participants in identifying an appropriate bidding strategy independent of a specific electricity market.

The remaining publications can be summarized as systems where multiple agents interact in an intelligent way [21]. [57] and [56] present automated negotiation protocols for trading between several local buyers and sellers, i.e., in a P2P market. [58] minimize the total transaction costs of micro-grid operators that trade with each other based on cooperative game theory.

The identified algorithms provide a solid overview of general algorithms for trading electricity. However, most of the identified algorithms only address day-ahead, intraday, or wholesale electricity markets without including the perspective of flexibly adjusting the planned electricity consumption schedule or providing flexibility services to the varying markets. Only [33], [38] and [42] consider prosumers that are able to provide flexibility to the grid from the supply as well as the demand side. Furthermore, several publications consider P2P market trading, i.e., [42], [45], [37], [57], [56], [50], and [47]. The possibility of trading small units of electricity on a local market enables the participating agents to provide flexibility. However, these publications focus on trading electricity without considering potential benefits and costs of, for example, shifting generation and load profiles. In summary, we identify only one publication where the authors incorporate flexibility in the trading algorithm in a specific form by developing optimal smart contracts for demand response on a blockchain-based platform for electric vehicles [53].

Accordingly, the results of our literature review illustrate that a lack of trading algorithms for flexibility exists. In particular, the identified papers lack the integration of the existing multiple markets for electricity and flexibility trading up to the actual physical delivery at the consumer's site, e.g., an energy-intensive production site. Hence, our work aims at closing this gap by developing an integrated ML framework for general flexibility trading.

\section{Algorithmic Framework}

In order to trade flexibility on several markets, a flexibility provider has to, first, define the quantity and time when flexibility can be provided. During 
this process, the flexibility provider has especially to consider the dependence between providing flexibility at a certain time interval and "recovering" from this adjustment of the planned baseline of generation or load [14]. Second, a flexibility provider has to evaluate a variety of possible markets where he*she can market a unit of his*her flexibility as well as the potential "consequences" and costs of adjusting the baseline profile. Finally, the provider has to evaluate, based on the expected profit and specific market restrictions, e.g., minimum bid requirements, on which market to actually place a bid. Our literature review illustrates that in order to handle the complexities and price volatility of different markets as well as to subsequently determine an appropriate trading strategy, tools within the AI environment seem appropriate. Based on the analyzed literature, we propose a ML- and NII-based integrated algorithmic framework for flexibility marketing. The objective of the proposed framework is to derive an optimal, multi-market trading strategy using a GA as a meta-learner, which relies on several market-level Q-learners to provide market-specific bidding strategies. Furthermore, we aim at including the interdependencies of baseline adjustments and different time horizons of markets by applying two different time intervals, i.e., a smaller time horizon for the market-level Q-learners compared to the GA meta-learner. Figure 3 depicts our proposed integrated framework.

The framework rests on a set of RL algorithms that learn optimal bidding strategies for different markets (i.e., spot markets, balancing markets, or local flexibility markets) where flexibility can be traded. During the learning process, the individual RL algorithms interact with the respective market environment by experimenting with different actions $a \in A$ (i.e., bidding strategies) and the corresponding transitions to a new state $s$ that is associated with a certain reward $r$. Whenever an action results in improvements (i.e., higher reward), the considered action is reinforced. In such a learning process, the RL agents are searching for the bidding strategy, through which the selected actions will bring the highest, long-term accumulated rewards.

For solving the RL problem, we propose a set of Q-learning algorithms for the different electricity and flexibility markets. Analogous to the RL problem, Q-learners receive market signals that link action-selection to market states and individual rewards. At each time step $t$, the Q-learner observes the current market state $s_{t}$. Accordingly, the Q-learner follows a certain bidding strategy $a_{t}$. As a result, the Q-learner then transitions to a new state $s_{t+1}$ and receives the corresponding reward $r_{t+1}$, i.e., the revenue from activating the submitted flexibility quantity minus the

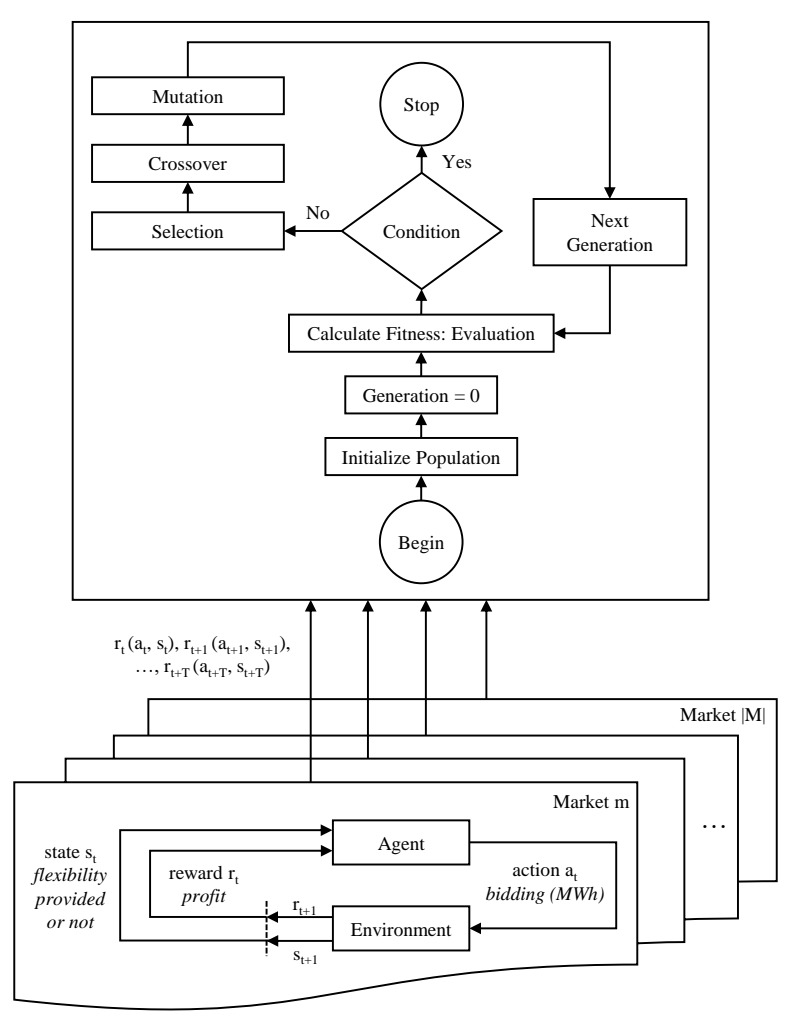

Figure 3: Integrated ML framework for flexibility trading

costs of the "inconvenience" and efficiency loss due to the adjustment of the baseline generation or load. During the learning process, the Q-learner gathers the corresponding state-action pairs in an expected value function that are stored in the so-called Q-table. With this table, the Q-learner is continuously searching for the best bidding strategy on the respective electricity or flexibility market. Thus, each individual Q-learner aims at maximizing Q-values and, thus, the expected reward from the respective bidding strategies. In this sense, the proposed Q-learners follow the following logic, proposed by [59]:

$$
Q_{i}^{\text {new }}=(1-\alpha) Q_{i}\left(s, a_{i}\right)+\alpha\left[\pi_{i}+\gamma \max Q_{i}\left(s^{\prime}, a_{i}\right]\right.
$$

The above logic describes, how Q-learners update the Q-table at each time step. The learning rate $\alpha$, taking values between 0 and 1 , describes, how much weight the Q-learner attributes to recent experiences (e.g., rewards). The discount rate $\gamma$, which also takes values between 0 and 1 , indicates, how foresighted Q-learners behave, i.e., how much they value future payoffs. When updating the Q-table, Q-learners face the trade-off between exploiting current rewards or choosing a seemingly inferior action $a_{t+1}$ for the sake 
of learning. For tackling this trade-off, we propose an $\epsilon$-greedy policy that chooses the action with the maximum Q-value with a probability $1-\epsilon$ and one of the remaining actions with the probability $\epsilon \geq 0$, regardless of their Q-value. This allows agents to experiment with different bidding strategies, while still maintaining a certain level of maximum returns.

Then, we propose the deployment of a meta-learner to derive the optimal combination of bidding strategies on the previously chosen electricity or flexibility markets. Given our analysis in Section 4, nature-inspired search algorithms are a suitable approach for finding an integrated optimal trading strategy. Consequently, we include a GA in our algorithmic framework to identify an optimal trading strategy for flexibility. Figure 3 depicts the process of applying the GA in our framework. Our approach of using a meta-learner in order to combine the optimal bidding strategies of different electricity markets not only allows us to consider different market requirements like, e.g., minimum bid size, but also to account for the time-dependence of providing flexibility. Especially in the case of a prosumer, which can provide flexibility on the supply as well as on the demand side, it is important to integrate the bidding strategies over several time intervals.

In order to initialize our population, we first have to encode the results of our individual Q-learning agents. Therefore, we aggregate the individual bidding actions $a_{t}$, states $s_{t}$, and corresponding rewards $r_{t}$ until $(t+$ $|T|)$ with $T$ being the entire calculation period of the GA meta-learner. To account for the time-dependency of providing flexibility, we apply the GA for every $\theta$, where $\theta$ represents a time interval from $t$ until $t+|T|$. Consequently, the local Q-learners could, for example, consider hourly time intervals for deriving the bidding strategy on the respective electricity or flexibility markets. The GA would then aggregate the individual hourly bids into daily bid functions. Following this time-aggregation from the Q-learning results of each market, the encoding of our individual strings represent the share of available flexibility in $\theta$ that is submitted to the respective electricity and flexibility market. Thus, the number of genes of each individual string in our population equals the number of considered electricity markets. $C=\{0,0.25,0.15,0.6\}$ represents an exemplary string where the trader would bid for nothing on the day-ahead market - see the first entry of $C-, 25 \%$ of the available flexibility on the intraday market, $15 \%$ on a real-time balancing-market, and $60 \%$ on a secondary frequency market following the pre-learned bidding course for $\theta$. After the encoding of the individual chromosomes, the population size for the initialization of the population has to be determined having the trade-off between computational time and optimality in mind. Hence, the decision on population size also depends on the number of electricity markets that are included and, thus, partly on the length of each individual string.

The fitness function represents the solution value to the addressed problem. Consequently, the fitness value of the best overall trading strategy represents the reward for the activated flexibility from the considered electricity markets. Hence, our fitness function aggregates the individual rewards $r_{t}$ determined by the individual strings corresponding to the bidding strategy pre-defined by actions $a_{t}$ and states $s_{t}$ over $t$ to $(t+|T|)$. Depending on the objectives of the application of the framework, the fitness function could be adjusted by adding risk preferences to the function.

For selecting the individuals of the population for the crossover and mutation process, various selection methods may be applied. The Roulette Wheel selection is a classic selection method where the individuals in the population are selected by randomly "spinning" the wheel of individuals. The probability that the selection will result in a particular string is calculated by the ratio of the fitness value of the individual to the total fitness of the population. However, often an alternative selection method, i.e., Tournament selection, converges faster than selection methods based on probability or rank [61]. With the Tournament selection, the genetic operator randomly selects two or more individuals that compete with each other based on their fitness value and the winning individual is added to the mating pool for reproduction. The reproduction includes a crossover and, if applied, a mutation function. For crossover, several crossover functions, e.g., single-site, two-site, or uniform crossover, are possible [62]. Depending on the case, applying mutation and the mutation rate may have positive or negative effects on the convergence of the GA, see for example [63]. As evaluating these hyper-parameters goes beyond the scope of this publication, we refer to further research to analyze the optimal hyper-parameter setting for our framework given a specific electricity market selection.

After the reproduction process is completed, the new generation of the population is evaluated given the fitness function. This process is iterated until a specific termination condition is met. In order to reduce computational time, we propose choosing a specific number of generations after which the GA is terminated. With the combination of the learned market bidding strategies, our framework allows identifying flexibility trading strategies on multiple markets, i.e., electricity markets like a day-ahead market as well as flexibility 
markets like a balancing market.

\section{Conclusion}

To conclude, flexibility trading is indispensable for the resilience of the future electricity system. As flexibility can be used for different applications such as, e.g., congestion management or frequency reserve, and consequently be traded on different markets, an optimal multi-market trading strategy is needed. In this paper, following a literature review on algorithmic approaches trading electricity and flexibility on corresponding markets, we provide a classification of these existing approaches. Additionally, we successfully achieved our objective to provide an algorithmic framework that learns a profit-maximizing strategy by simultaneously trading different flexibility products in a multi-market setting. Consequentially, when implementing our framework, a flexibility provider can consider multiple markets, where the flexibility provider wants to offer flexibility. The application of the framework can provide incentives to leverage flexibility potentials within the electricity system to address the challenges presented by a rising penetration of renewable energies. Furthermore, the implementation of our AI framework may help to increase the resilience of the electricity system by increasing the overall amount of traded flexibility. However, the range of potential trading strategies depend on the characteristics of the markets relevant in the respective environment and the respective market design, e.g., existence of regionally resolved price signals (financial incentives) and market entry barriers.

Regarding further research, we aim to provide a "reality check" using expert interviews with interview partners from the application side of flexibility trading algorithms from GENCOs, large electricity suppliers, municipal utility companies, and specialized traders. Based on these expert interviews, we further aim to implement the proposed framework using historical electricity market data, e.g., EPEX SPOT day-ahead prices, and obtained data regarding demand side flexibility from companies within the Kopernikus-Project SynErgie to evaluate the benefits of multi-market flexibility trading. Further, the framework can also be used to minimize the risk of flexibility trading by optimizing the trade over different markets, time horizons, and delivery times. Therefore, a risk portfolio management perspective can be added to the framework in future research using existing literature, e.g., [64] and [65].

\section{Acknowledgments}

The authors gratefully acknowledge the financial support of the Kopernikus-Project "SynErgie" by the Federal Ministry of Education and Research of Germany (BMBF) and the project supervision by the project management organization Projektträger Jülich (PtJ).

The authors thank the Graduate School of the Bavarian Center for Battery Technology (BayBatt) for ongoing support.

\section{References}

[1] C. Zöphel, S. Schreiber, T. Müller, and D. Möst, "Which flexibility options facilitate the integration of intermittent renewable energy sources in electricity systems?," Current Sustainable/Renewable Energy Reports, vol. 5, no. 1, pp. 37-44, 2018.

[2] G. Papaefthymiou, E. Haesen, and T. Sach, "Power system flexibility tracker: Indicators to track flexibility progress towards high-res systems," Renewable energy, vol. 127, pp. 1026-1035, 2018.

[3] S. Zhang, D. May, P. Atrazhev, M. Gul, A. Leach, T. Weis, and P. Musilek, "Etx: A flexible simulation framework for design of transactive energy systems," in 2019 IEEE Canadian Conference of Electrical and Computer Engineering (CCECE), pp. 1-4, IEEE, 2019.

[4] M.-F. Körner, D. Bauer, R. Keller, M. Rösch, A. Schlereth, P. Simon, T. Bauernhansl, G. Fridgen, and G. Reinhart, "Extending the automation pyramid for industrial demand response," Procedia CIRP, vol. 81, pp. 998-1003, 2019.

[5] C. Vizjak, "The role of algorithms in energy trading." Retrieved May 20, 2021, from https://www.trayport.com/blog/ the-role-of-algorithms-in-energyItrading/, 2020.

[6] L. Malceniece, K. Malcenieks, and T. J. Putniņš, "High frequency trading and comovement in financial markets," Journal of Financial Economics, vol. 134, no. 2, pp. 381-399, 2019.

[7] A. Esmat, P. Pinson, and J. Usaola, "Decision support program for congestion management using demand side flexibility," in 2017 IEEE Manchester PowerTech, pp. 1-6, IEEE, 2017.

[8] S. Ø. Ottesen, A. Tomasgard, and S.-E. Fleten, "Multi market bidding strategies for demand side flexibility aggregators in electricity markets," Energy, vol. 149, pp. 120-134, 2018.

[9] M. Badami, G. Fambri, S. Mancò, M. Martino, I. G. Damousis, D. Agtzidis, and D. Tzovaras, "A decision support system tool to manage the flexibility in renewable energy-based power systems," Energies, vol. 13, no. 1, p. 153, 2020.

[10] O. M. Sedeh and B. Ostadi, "Optimization of bidding strategy in the day-ahead market by consideration of seasonality trend of the market spot price," Energy Policy, vol. 145, p. 111740, 2020.

[11] A. Y. R. González, M. P. Alonso, F. Lezama, L. Rodríguez, E. M. de Cote, E. F. Morales, L. E. Sucar, and D. D. Crockett, "A competitive and profitable multi-agent autonomous broker for energy markets," Sustainable Cities and Society, vol. 49, p. 101590, 2019. 
[12] S. M. A. N. Javareshk, S. A. K. Biyouki, and S. H. Darban, "Optimal bidding strategy in double-sided auctions of the competitive power market using agent-based algorithm," in 2019 27th Iranian Conference on Electrical Engineering (ICEE), pp. 798-803, IEEE, 2019.

[13] A. A. Novirdoust, M. Bichler, C. Bojung, H. U. Buhl, G. Fridgen, V. Gretschko, L. Hanny, J. Knörr, F. Maldonado, K. Neuhoff, C. Neumann, M. Ott, J. C. Richstein, M. Rinck, M. Schöpf, P. Schott, A. Sitzmann, J. Wagner, J. Wagner, and M. Weibelzahl, "Electricity spot market design 2030-2050," Februar 2021.

[14] P. Schott, J. Sedlmeir, N. Strobel, T. Weber, G. Fridgen, and E. Abele, "A generic data model for describing flexibility in power markets," Energies, vol. 12, Mai 2019.

[15] R. J. Heffron, M.-F. Körner, M. Schöpf, J. Wagner, and M. Weibelzahl, "The role of flexibility in the light of the covid-19 pandemic and beyond: Contributing to a sustainable and resilient energy future in europe," Renewable and Sustainable Energy Reviews, vol. 140, p. 110743, 2021

[16] H. Kondziella and T. Bruckner, "Flexibility requirements of renewable energy based electricity systems - a review of research results and methodologies," Renewable and Sustainable Energy Reviews, vol. 53, pp. 10-22, 2016.

[17] R. Heffron, M.-F. Körner, J. Wagner, M. Weibelzahl, and G. Fridgen, "Industrial demand-side flexibility: A key element of a just energy transition and industrial development," Applied Energy, vol. 269, p. 115026, 2020.

[18] J. Villar, R. Bessa, and M. Matos, "Flexibility products and markets: Literature review," Electric Power Systems Research, vol. 154, pp. 329-340, 2018

[19] J. Webster and R. T. Watson, "Analyzing the past to prepare for the future: Writing a literature review," MIS quarterly, pp. xiii-xxiii, 2002.

[20] J. Vom Brocke, A. Simons, K. Riemer, B. Niehaves, R. Plattfaut, and A. Cleven, "Standing on the shoulders of giants: Challenges and recommendations of literature search in information systems research," Communications of the association for information systems, vol. 37, no. 1, p. 9, 2015 .

[21] I. Antonopoulos, V. Robu, B. Couraud, D. Kirli, S. Norbu, A. Kiprakis, D. Flynn, S. Elizondo-Gonzalez, and S. Wattam, "Artificial intelligence and machine learning approaches to energy demand-side response: A systematic review," Renewable and Sustainable Energy Reviews, vol. 130, p. 109899, 2020.

[22] E. Dall'Anese, P. Mancarella, and A. Monti, "Unlocking flexibility: Integrated optimization and control of multienergy systems," IEEE Power and Energy Magazine, vol. 15, no. 1, pp. 43-52, 2017.

[23] R. Moreno, A. Street, J. M. Arroyo, and P. Mancarella, "Planning low-carbon electricity systems under uncertainty considering operational flexibility and smart grid technologies," Philosophical Transactions of the Royal Society A: Mathematical, Physical and Engineering Sciences, vol. 375, no. 2100, p. 20160305 , 2017.

[24] H. Golmohamadi, K. G. Larsen, P. G. Jensen, and I. R. Hasrat, "Optimization of power-to-heat flexibility for residential buildings in response to day-ahead electricity price," Energy and Buildings, vol. 232, p. 110665, 2021.
[25] C. Gomes, "Artificial intelligence and operations research: Challenges and opportunities in planning and scheduling," The Knowledge Engineering Review, vol. 15, pp. 1-10, 032000 .

[26] A. Tiguercha, A. Ladjici, M. Boudour, and M. Hazli, "Day ahead a electricity market analysis through a neuroevolution algorithm," in 2016 IEEE International Energy Conference (ENERGYCON), pp. 1-6, IEEE, 2016.

[27] T. Pinto, T. M. Sousa, H. Morais, I. Praça, and Z. Vale, "Metalearning to support competitive electricity market players' strategic bidding," Electric Power Systems Research, vol. 135, pp. 27-34, 2016.

[28] C. Monteiro, L. A. Fernandez-Jimenez, and I. J. Ramirez-Rosado, "Predictive trading strategy for physical electricity futures," Energies, vol. 13, no. 14, p. 3555, 2020.

[29] W. Wang and N. Yu, "A machine learning framework for algorithmic trading with virtual bids in electricity markets," in 2019 IEEE Power \& Energy Society General Meeting (PESGM), pp. 1-5, IEEE, 2019.

[30] N. Wang, W. Xu, W. Shao, and Z. Xu, "A q-cube framework of reinforcement learning algorithm for continuous double auction among microgrids," Energies, vol. 12, no. 15, p. 2891, 2019.

[31] G. Yu, H. Zhang, P. Yang, and Z. Chen, "Bidding model of generation company considering volatility of futures market," in 2020 12th IEEE PES Asia-Pacific Power and Energy Engineering Conference (APPEEC), pp. 1-5, IEEE, 2020.

[32] X. Wang, H. Chen, J. Wu, Y. Ding, Q. Lou, and S. Liu, "Bi-level multi-agents interactive decision-making model in regional integrated energy system," in 2019 IEEE 3rd Conference on Energy Internet and Energy System Integration (EI2), pp. 2103-2108, IEEE, 2019.

[33] I. Kalysh, A. Alimkhan, I. Temirtayev, H. K. Nunna, S. Doolla, and K. Vipin, "Dynamic programming based peer-to-peer energy trading framework for smart microgrids," in 2019 IEEE 13th International Conference on Compatibility, Power Electronics and Power Engineering (CPE-POWERENG), pp. 1-6, IEEE, 2019.

[34] S. Baltaoglu, L. Tong, and Q. Zhao, "Algorithmic bidding for virtual trading in electricity markets," IEEE Transactions on Power Systems, vol. 34, no. 1, pp. 535-543, 2018.

[35] H. Chen, R. Bo, R. Das, and D. Wunsch, "Exploring reinforcement learning method in bidding strategy development for day-ahead electricity market," in 2020 12th IEEE PES Asia-Pacific Power and Energy Engineering Conference (APPEEC), pp. 1-5, IEEE, 2020.

[36] N. Mazzi and P. Pinson, "Purely data-driven approaches to trading of renewable energy generation," in 2016 13th International Conference on the European Energy Market (EEM), pp. 1-5, IEEE, 2016.

[37] S. Bose, E. Kremers, E. M. Mengelkamp, J. Eberbach, and C. Weinhardt, "Reinforcement learning in local energy markets," Energy Informatics, vol. 4, no. 1, pp. 1-21, 2021.

[38] G. Bertrand and A. Papavasiliou, "Reinforcement-learning based threshold policies for continuous intraday electricity market trading," in 2019 IEEE Power \& Energy Society General Meeting (PESGM), pp. 1-5, IEEE, 2019. 
[39] K. Purushothaman and V. Chandrakala, "Roth-erev reinforcement learning approach for smart generator bidding towards long term electricity market operation using agent based dynamic modeling," Electric Power Components and Systems, vol. 48, no. 3, pp. 256-267, 2020.

[40] S. M. A. Naseri-Javareshk, S. H. Darban, A. Noori, and S. A. Kouche-Biyouki, "Using eligibility traces in bidding strategy of gencos in spinning reserve and energy markets for different market allocation models," in Electrical Engineering (ICEE), Iranian Conference on, pp. 1350-1355, IEEE, 2018.

[41] Y. Liang, C. Guo, Z. Ding, and H. Hua, "Agent-based modeling in electricity market using deep deterministic policy gradient algorithm," IEEE Transactions on Power Systems, vol. 35, no. 6, pp. 4180-4192, 2020.

[42] J.-G. Kim and B. Lee, "Automatic p2p energy trading model based on reinforcement learning using long short-term delayed reward," Energies, vol. 13, no. 20, p. 5359,2020 .

[43] D. Cao, W. Hu, X. Xu, T. Dragičević, Q. Huang, Z. Liu, Z. Chen, and F. Blaabjerg, "Bidding strategy for trading wind energy and purchasing reserve of wind power producer-a drl based approach," International Journal of Electrical Power \& Energy Systems, vol. 117, p. 105648, 2020

[44] Y. Xu, L. Yu, G. Bi, M. Zhang, and C. Shen, "Deep reinforcement learning and blockchain for peer-to-peer energy trading among microgrids," in 2020 International Conferences on Internet of Things (iThings) and IEEE Green Computing and Communications (GreenCom) and IEEE Cyber, Physical and Social Computing (CPSCom) and IEEE Smart Data (SmartData) and IEEE Congress on Cybermatics (Cybermatics), pp. 360-365, IEEE, 2020.

[45] T. Chen and S. Bu, "Realistic peer-to-peer energy trading model for microgrids using deep reinforcement learning," in 2019 IEEE PES Innovative Smart Grid Technologies Europe (ISGT-Europe), pp. 1-5, IEEE, 2019.

[46] G. Sun, X. Wang, L. Yang, B. Ma, L. He, and R. Zhang, "A method for power suppliers' optimal cooperative bidding strategies considering network losses," Global Energy Interconnection, vol. 3, no. 4, pp. 335-345, 2020.

[47] F. Lezama, J. Soares, R. Faia, Z. Vale, O. Kilkki, S. Repo, and J. Segerstam, "Bidding in local electricity markets with cascading wholesale market integration," International Journal of Electrical Power \& Energy Systems, vol. 131, p. 107045, 2021.

[48] S. Singh, M. Fozdar, and A. K. Singh, "Coordinating bidding strategy of profit maximization for competitive power suppliers in energy and reserve markets," in 2018 8th IEEE India International Conference on Power Electronics (IICPE), pp. 1-6, IEEE, 2018.

[49] Y. Gao, X. Zhou, J. Ren, X. Wang, and D. Li, "Double layer dynamic game bidding mechanism based on multi-agent technology for virtual power plant and internal distributed energy resource," Energies, vol. 11, no. 11, p. 3072, 2018.

[50] F. Lezama, J. Soares, R. Faia, P. Faria, and Z. Vale, "Learning bidding strategies in local electricity markets using a nature-inspired algorithm," in 2020 17th International Conference on the European Energy Market (EEM), pp. 1-6, IEEE, 2020.
[51] F. Lezama, J. Soares, and Z. Vale, "Optimal bidding in local energy markets using evolutionary computation," in 2019 20th International Conference on Intelligent System Application to Power Systems (ISAP), pp. 1-6, IEEE, 2019.

[52] D. Shah and S. Chatterjee, “Optimal genco's bidding strategy in a power exchange facilitating combined power and emission trading using intelligent programmed genetic algorithm," International Transactions on Electrical Energy Systems, vol. 30, no. 8, p. e12463, 2020.

[53] B. Wang, W. Liu, M. Wang, and W. Shen, "Research on bidding mechanism for power grid with electric vehicles based on smart contract technology," Energies, vol. 13, no. 2, p. 390, 2020.

[54] V. M. S. Reddy, B. Subramanyam, and M. S. Kalavathi, "Bidding strategy in south region day-ahead demand model by improved artificial bee colony algorithm," International Journal of Power and Energy Conversion, vol. 8, no. 2, pp. 204-224, 2017.

[55] K. Dehghanpour, M. H. Nehrir, J. W. Sheppard, and N. C. Kelly, "Agent-based modeling in electrical energy markets using dynamic bayesian networks," IEEE Transactions on Power Systems, vol. 31, no. 6, pp. 4744-4754, 2016.

[56] C. Etukudor, B. Couraud, V. Robu, W.-G. Früh, D. Flynn, and C. Okereke, "Automated negotiation for peer-to-peer electricity trading in local energy markets," Energies, vol. 13, no. 4, p. 920, 2020.

[57] C. Etukudor, V. Robu, B. Couraud, G. Kocher, W.-G. Früh, D. Flynn, and C. Okereke, "Automated negotiation for peer-to-peer trading of renewable energy in off-grid communities," in 2019 IEEE PES/IAS PowerAfrica, pp. 1-6, IEEE, 2019.

[58] J. Ni and Q. Ai, "Economic power transaction using coalitional game strategy in micro-grids," IET Generation, Transmission \& Distribution, vol. 10, no. 1, pp. 10-18, 2016.

[59] R. S. Sutton and A. G. Barto, Reinforcement learning: An introduction. MIT press, 2018.

[60] Z. Beheshti and S. M. H. Shamsuddin, "A review of population-based meta-heuristic algorithms," Int. J. Adv. Soft Comput. Appl, vol. 5, no. 1, pp. 1-35, 2013.

[61] J. Zhong, X. Hu, J. Zhang, and M. Gu, "Comparison of performance between different selection strategies on simple genetic algorithms," in International conference on computational intelligence for modelling, control and automation and international conference on intelligent agents, web technologies and internet commerce (CIMCA-IAWTIC'06), vol. 2, pp. 1115-1121, IEEE, 2005.

[62] G. C. Onwubolu and B. Babu, New optimization techniques in engineering, vol. 141. Springer, 2013.

[63] S. Lim and M. Kuby, "Heuristic algorithms for siting alternative-fuel stations using the flow-refueling location model," European Journal of Operational Research, vol. 204, no. 1, pp. 51-61, 2010.

[64] J. Kettunen, A. Salo, and D. W. Bunn, "Optimization of electricity retailer's contract portfolio subject to risk preferences," IEEE Transactions on Power Systems, vol. 25, no. 1, pp. 117-128, 2010.

[65] M. E. Mangram, "A Simplified Perspective Of The Markowitz Portfolio Theory," Global Journal of Business Research, vol. 7, no. 1, pp. 59-70, 2013. 